\title{
Relação entre uso do esquema antirretroviral e aterosclerose coronariana em pacientes HIV positivos no estado de Sergipe
}

\author{
Relationship between antiretroviral regimen use and coronary atherosclerosis in HIV-positive \\ patients in the state of Sergipe \\ Relación entre el uso de régimen antirretroviral y la aterosclerosis coronaria en pacientes VIH \\ positivos en el estado de Sergipe
}

Débora Kelly Santos de Oliveira ORCID: https://orcid.org/0000-0001-6261-9931 Secretaria Municipal da Saúde de Aracaju, Brasil E-mail: debkso42@gmail.com

João Eduardo Andrade Tavares de Aguiar ORCID: https://orcid.org/0000-0002-9576-8148 Universidade Federal de Sergipe, Brasil

E-mail: joaoeduardoandrade97@gmail.com

Alexia Ferreira Rodrigues

ORCID: https://orcid.org/0000-0002-8307-7040 Universidade Federal de Sergipe, Brasil E-mail: alexia.ferreira23@hotmail.com

Vinícius Fernando Alves Carvalho ORCID: https://orcid.org/0000-0003-0303-0996 Universidade Federal de Sergipe, Brasil E-mail: viniciusmedicinaufs@gmail.com

Joselina Luzia Menezes Oliveira ORCID: https://orcid.org/0000-0002-4325-0590 Universidade Federal de Sergipe, Brasil E-mail: joselinamenezes@gmail.com Antônio Carlos Sobral Sousa ORCID: https://orcid.org/0000-0002-4158-9726 Universidade Federal de Sergipe, Brasil E-mail: acssousa@terra.com.br

Dalmo Correia ORCID: https://orcid.org/0000-0002-2174-5058 Universidade Federal do Triângulo Mineiro, Brasil E-mail: dalmo@mednet.com.br Ângela Maria da Silva ORCID: https://orcid.org/0000-0001-9330-537X Universidade Federal de Sergipe, Brasil E-mail: angela.silva910@gmail.com

\begin{abstract}
Resumo
A terapia antirretroviral (TARV) coincide com a evidencia da ocorrência de doenças crônicas cardiovasculares de origem aterosclerótica, quando associada a uma terapia de longo prazo. O objetivo foi avaliar a relação entre aterosclerose e uso de esquema antirretroviral no tratamento de pacientes HIV positivo, e quantificar o escore de cálcio neste grupo, correlacionando-o com o tempo de uso da TARV. Trata-se de um estudo tranversal de pacientes adultos portadores de HIV/AIDS, selecionados de forma consecutiva e não-aleatória, assintomáticos do ponto de vista cardiovascular, provenientes dos dois serviços de referência em atendimento ambulatorial do estado de Sergipe, com idade maior que 18 anos e em uso de TARV. Da amostra, 65,4\% eram do sexo masculino e 34,6\% do sexo feminino, com idade média de 46 anos. A aterosclerose coronariana, detectada pelo escore de cálcio, foi encontrado em 24 pacientes $(31,17 \%)$. A duração da terapia antirretroviral se relacionou à aterosclerose e houve diferenças significativas entre os diferentes esquemas antirretrovirais. Além disso, os resultados mostram uma associação entre TARV contendo IP. A idade e o tempo de exposição ao uso da TARV foram significativos na presença de aterosclerose. Os valores de $\mathrm{p}$ foram considerados estatisticamente significativos em $100 \%$ das variáveis analisadas e o desafio é promover a prevenção de riscos cardiovasculares para reduzir o número de eventos coronarianos, e o perfil traçado com esta coleta permitirá uma abordagem mais criteriosa desses pacientes, sobretudo no caráter preventivo com o cuidado dos pacientes portadores de HIV/AIDS no estado de Sergipe.
\end{abstract}


Palavras-chave: Doença da artéria coronariana; Terapia antirretroviral de alta atividade; HIV.

\begin{abstract}
Antiretroviral therapy (ART) coincides with the evidence of occurrence of chronic cardiovascular diseases of atherosclerotic origin, when associated with long-term therapy. The objective was to evaluate the relationship between atherosclerosis and the antiretroviral regimen use in the treatment of HIV-positive patients and to quantify the calcium score in this group, correlating it with the duration of ART use. This was a cross-sectional study of adult patients with HIV/AIDS, consecutively and non-randomly selected, asymptomatic from the cardiovascular point of view, from two reference services in outpatient care in the state of Sergipe, aged over 18 years and using antiretroviral therapy. Of the sample, $65.4 \%$ were male and $34.6 \%$ were female, with a mean age of 46 years. Coronary atherosclerosis, detected by the calcium score, was found in 24 patients $(31.17 \%)$. The duration of antiretroviral therapy was related to atherosclerosis and there were significant differences between the different antiretroviral regimens. In addition, the results show an association between ART containing a protease inhibitor. Age and exposure time to ART use were significant in the presence of atherosclerosis. The $\mathrm{p}$ values were considered statistically significant in $100 \%$ of the variables analyzed and the challenge is to promote the prevention of cardiovascular risks to reduce the number of coronary events, and the profile traced with this collection will allow a more careful approach to these patients, especially in the character preventive care with HIV/AIDS patients in the state of Sergipe.
\end{abstract}

Keywords: Coronary artery disease; Highly active antiretroviral therapy; HIV.

\begin{abstract}
Resumen
La terapia antirretroviral (TAR) coincide con la evidencia de ocurrencia de enfermedades cardiovasculares crónicas de origen aterosclerótico, cuando se asocia a terapia a largo plazo. El objetivo fue evaluar la relación entre aterosclerosis y uso del régimen antirretroviral en el tratamiento de pacientes VIH positivos y cuantificar el puntaje de calcio en este grupo, correlacionándolo con la duración del uso de TARV. Se trata de un estudio transversal de pacientes adultos con VIH/SIDA, seleccionados de forma consecutiva y no aleatoria, asintomáticos desde el punto de vista cardiovascular, de dos servicios de referencia en atención ambulatoria de Sergipe, mayores de 18 años y en uso de antirretrovirales. De la muestra, el 65,4\% eran hombres y el 34,6\% mujeres, con edad media de 46 años. La aterosclerosis coronaria, detectada por el puntaje de calcio, se encontró en 24 pacientes $(31,17 \%)$. La duración de la terapia antirretroviral se relacionó con aterosclerosis y hubo diferencias significativas entre los diferentes regímenes antirretrovirales. Además, los resultados muestran una asociación entre los TAR que contienen un inhibidor de la proteasa. La edad y el tiempo de exposición al uso de TAR fueron significativos en presencia de aterosclerosis. Los valores de p fueron considerados estadísticamente significativos en el $100 \%$ de las variables analizadas y el reto es promover la prevención de riesgos cardiovasculares para reducir el número de eventos coronarios, y el perfil trazado con esta recopilación permitirá un abordaje más cuidadoso de estos pacientes, especialmente en el carácter de atención preventiva a pacientes con VIH/SIDA en Sergipe.
\end{abstract}

Palabras clave: Enfermedad de la arteria coronaria; Terapia antirretroviral altamente activa; VIH.

\title{
1. Introdução
}

Diariamente, 14 mil pessoas são infectadas pelo vírus da imunodeficiência humana (HIV) e desde sua descoberta, a epidemia já gerou quase 30 milhões de óbitos pelo mundo (WHO, 2016). Estima-se atualmente que a infecção acometa 42 milhões de pessoas no mundo, sendo na América Latina 1,8 milhões de infectados. O Brasil, entre os países desta região, é o mais afetado em números absolutos, com aproximadamente 1,2 milhões (Thienemann, Sliwa \& Rockstroh, 2013). Registra-se ainda 228.988 mil casos no Nordeste, 8936 casos em Sergipe e 7566 casos em Aracaju (Brasil, 2020).

O tratamento adquiriu grandes avanços após a introdução da terapia antirretroviral potente (TARV) propiciando redução da mortalidade e morbidade associados à doença, até então, onde o prognóstico dependia do diagnóstico precoce e tratamento das infecções oportunistas. Estima-se que, desde 1995, um total de 2,5 milhões de mortes foram evitadas em países de baixa e média renda, principalmente devido ao acesso crescente à terapia antirretroviral (UNAIDS, 2010; UNAIDS, 2011).

A TARV coincide com a mudança no espectro da doença, aumento da expectativa de vida dos pacientes e evidenciase, contudo, a ocorrência de doenças crônicas na frequência de doenças cardiovasculares de origem aterosclerótica. Considerando a Doença Arterial Coronariana (DAC) causa importante de morbimortalidade na população de portadores de HIV e seu aparecimento decorrente da infecção viral crônica e/ou efeitos colaterais dos antirretrovirais que podem resultar em distúrbios metabólicos, é possível que a quantificação da calcificação coronária nos pacientes portadores de HIV acrescente dados relevantes para a estratificação de risco da DAC, viabilizando medidas preventivas nesta população (Saddi \& Giraldez, 
2009).

Justifica-se a importância deste estudo pela relevância do ponto de vista científico uma vez que trazem contribuição ao conhecimento, preenchendo uma lacuna existente no que está associado à aterosclerose coronária nos pacientes HIV positivos e do ponto de vista socioeconômico contribuindo na sobrevida destes pacientes, com redução de eventos cardiovasculares a partir de um diagnóstico precoce atuando de forma preventiva, o que reduz custos com internações e procedimentos.

Assim, o objetivo deste estudo foi avaliar a relação entre aterosclerose e uso de esquema antirretroviral no tratamento de pacientes HIV positivo, quantificar o escore de cálcio (EC) nos pacientes HIV positivos em tratamento com antirretroviral e correlacionando-o com o tempo de uso da TARV.

\section{Metodologia}

A investigação foi conduzida pelo pesquisador principal em face ao seu mestrado e fez parte de um grande projeto prospectivo, transversal, desenvolvido pela Universidade Federal de Sergipe capitaneado por pesquisadores que tem fomento em trabalhos científicos em Cardiologia e Infectologia.

\subsection{Tipo de estudo}

Estudo observacional, transversal e analítico.

\subsection{População de referência}

Os pacientes HIV/AIDS que participaram deste estudo foram selecionados de forma consecutiva e não-aleatória, assintomáticos do ponto de vista cardiovascular, provenientes dos dois serviços de referência em atendimento ambulatorial do estado de Sergipe: o Centro de Especialidades Médicas de Aracaju (CEMAR) e o Hospital Universitário da Universidade Federal de Sergipe (HU-UFS) com idade maior de 18 anos e em uso de TARV. Para a estimativa do tamanho da amostra considerou-se o intervalo de confiança de $95 \%$ e uma diferença aceitável de cerca de $10 \%$. Os indivíduos foram convidados a participar de forma voluntária do presente trabalho.

\subsection{Revisão de prontuários e de registros de dispensação de antirretrovirais}

Para a composição da amostra deste estudo foram selecionados os pacientes que realizavam o tratamento no período de Agosto de 2018 a Junho de 2019 e atendiam os critérios de elegibilidade. A partir da amostra selecionada, a análise buscou dados como:

1. Análise retrospectiva dos prontuários clínicos dos participantes: coleta da data do diagnóstico da infecção pelo HIV, dados referentes à categoria de exposição, data do primeiro atendimento na instituição, data do início da TARV, dados laboratoriais (colesterol total e frações, triglicérides, glicemia), dados de exames de quantificação da carga viral (valores absolutos e respectivos valores em logarítmicos) e da contagem de linfócito T CD4 e CD8 (valores absolutos e percentuais).

2. Análise retrospectiva da TARV dos participantes: drogas utilizadas com data de início e término que serão colhidos dos registros de dispensação, da Unidade Dispensadora de Medicamentos (UDM) dos serviços de referência, por membros da equipe, utilizando relatório padronizado do Sistema de Controle Logístico de Medicamentos (SICLOM).

\subsection{Critérios de inclusão}

Foram incluídos pacientes homens ou mulheres, com idade igual ou superior a 18 anos, soropositivos para HIV, com capacidade para entender e disposição (deles ou dos responsáveis) de assinar o Termo de Consentimento Livre e Esclarecido 
(TCLE).

\subsection{Critérios de exclusão}

Foram excluídos pacientes sintomáticos do ponto de vista cardiovascular e pacientes com DAC estabelecida (DAC prévia), assim como aqueles com sinais clínicos de infecções ativas, com internamento recente, pacientes debilitados e gestantes.

\subsection{Aspectos éticos}

O estudo, em consonância com a linha de pesquisa, faz parte de um braço de um estudo maior e é um corte transversal de um estudo longitudinal e prospectivo que foi conduzido por pesquisadores da Universidade Federal de Sergipe e aprovado pelo comitê de ética, seguindo as premissas da Resolução 466/12/CONEPE, sob o CAAE 70279317.0.0000.5546 e Parecer de número 2.244.171. Os pacientes, após esclarecimento dos objetivos do estudo, confirmaram sua aceitação em participar da pesquisa por meio da assinatura de TCLE. Em atendimento ao preconizado na Resolução 196/96, do Conselho Nacional de Saúde, foram preservados o anonimato dos pacientes e o sigilo das informações, de modo que, pela leitura do material não foi possível a identificação de nenhum paciente. Dados e amostras dos pacientes foram codificadas com acesso apenas pelo pesquisador principal. O comitê de ética seria informado em caso de interrupção do estudo e a publicação dos dados em revista de comunicação científica sem a identificação dos pacientes.

\subsection{Análise dos riscos}

A pesquisa foi considerada com riscos mínimos para os envolvidos no estudo, com exames não invasivos e bem tolerados. A anamnese, análise dos prontuários e os exames laboratoriais foram avaliações que já faziam parte da rotina do CEMAR e do HU-UFS.

\subsection{Análise dos benefícios}

Os achados do estudo foram importantes na detecção precoce de aterosclerose coronariana, possibilitando à implementação de medidas preventivas de eventos coronarianos agudos, controle mais rigoroso de fatores de risco e adesão dos pacientes ao tratamento, bem como um incremento do risco estimado pelo EC pré e pós TARV, assim como a influência do tempo de tratamento, da idade e do sexo. A comunidade, em especial os pacientes HIV positivos se beneficiam nestes aspectos.

\subsection{Procedimentos}

Os voluntários responderam ao questionário e, após, foi coletado o sangue para exames hematológicos e bioquímicos e encaminhados ao cardiologista. Foram coletadas amostras de sangue dos pacientes incluídos na pesquisa, após cerca de 12 horas de jejum. As medidas foram de colesterol total e frações (LDL-C e HDL-C), triglicérides, glicemia, CD4 e carga viral em conformidade com especificações e métodos do Laboratório de Análises Clínicas do HU-UFS.

Fatores de risco considerados para efeitos de classificação do escore de Framingham foram analisados separadamente com base na importância entre eles como dislipidemia, triglicérides, colesterol total, hipertensão e tabagismo, além de sexo e idade.

A Estratificação clínica de risco coronariano foi realizada através da avaliação das características clínicas dos pacientes e coleta no questionário de fatores de risco coronarianos clássicos, assim como resultados de exames laboratoriais e medida da pressão arterial, agrupando-os conforme o Escore de Framingham. 
Os pacientes foram classificados como hipertensos aqueles que apresentaram pressão sistólica e diastólica superior a $140 \mathrm{mmHg}$ e a $90 \mathrm{mmHg}$, respectivamente, os parâmetros de resultados de triglicérides normal menor que $150 \mathrm{mg} / \mathrm{dl}$ e colesterol total normal menor que 190mg/dl de acordo com a Sociedade Brasileira de Cardiologia. A presença de diabetes mellitus foi considerada em pacientes com histórico de diabetes e uso de hipoglicemiantes orais ou insulina, bem como a constatação de glicemia plasmática em jejum superior a $126 \mathrm{mg} / \mathrm{dl}$ segundo critérios de definição pela Sociedade Brasileira de Diabetes.

Informações sobre o esquema antirretroviral foram obtidas a partir dos prontuários e utilizando relatório padronizado do SICLOM. Os pacientes foram classificados como "usuários" ou "não usuários" dos esquemas contendo inibidores de protease (IP). Pacientes que mudaram a TARV, mas permaneceram na mesma classe de fármacos não foram excluídos na classificação de usuários.

\subsection{Tratamento dos dados}

$\mathrm{Na}$ análise descritiva foi realizada para detalhar os resultados. Para as variáveis qualitativas os dados foram expressos em termos relativos (percentuais) e frequências absolutas $(\mathrm{N})$, e para as variáveis quantitativas pela média. Na análise estatística foram utilizados os testes: Qui-quadrado de aderência e Exato de Fisher e os valores de $\mathrm{p} \leq 0,05$ foram considerados estatisticamente significativos. Considerou-se ainda o intervalo de confiança de $95 \%$ e uma diferença aceitável de cerca de 10\%. Para as variáveis independentes (tratamento e tempo de uso) foi realizada análise não paramétrica.

A pesquisa foi exequível uma vez que foi realizada no atendimento ambulatorial dos pacientes HIV positivo, não configurando extrapolação extrema do que já faz parte do acompanhamento destes pacientes. Os pesquisadores possuíam plena capacidade de captação dos pacientes com HIV e os ambulatórios haviam cerca de 4.304 pacientes em acompanhamento no momento. O aporte de material humano, com pesquisadores dos grupos de pesquisa para tal atendimento nos ambulatórios assegura a sua exequibilidade. Tudo isso é relevante do ponto de vista científico uma vez que trazem real contribuição ao conhecimento, preenchendo uma lacuna existente no conhecimento daquilo que está associado à aterosclerose coronária nos pacientes HIV positivos.

\section{Resultados}

Participaram deste estudo 164 pacientes portadores do HIV/AIDS, selecionados de forma consecutiva e não-aleatória, assintomáticos do ponto de vista cardiovascular. Destes, 05 pacientes desistiram em algum momento da pesquisa. Dessa amostra, 23,9\% dos pacientes fizeram todos os exames de acompanhamento necessários para a análise da pesquisa, 59,1\% fizeram teste ergométrico (TE) e ecocardiograma (ECO) e apenas 43,9\% fizeram tomografia computadorizada (TC) cardíaca.

$\mathrm{Na}$ análise considerou-se uma prevalência de aterosclerose nos pacientes HIV positivos documentada pelo EC na literatura em torno de $40 \%$ (para os diversos níveis de calcificação coronária) e submetidos à TARV, acompanhados nos serviços ambulatoriais do estado estando em tratamento no período 32,1\% no CEMAR e 67,9\% no HU-UFS (Tabela 1).

Tabela 1. Pacientes HIV/AIDS em uso de TARV, acompanhados nos serviços ambulatoriais, Aracaju, 2018.

\begin{tabular}{ccccc}
\hline Serviço & Total & \% & Valor de p \\
\hline CEMAR & 51 & $32,1 \%$ & $<0,01$ \\
HU-UFS & 108 & $67,9 \%$ & $\mathbf{1 0 0 \%}$ \\
\hline Total & $\mathbf{1 5 9}$ & \\
\hline
\end{tabular}

Fonte: SICLOM/PMIST-AIDS-HV/REPVAS/DVAS/SMS-AJU. 
A idade dos pacientes variou entre 19 e 80 anos, com idade média de 46 anos. A idade tem influência direta no desenvolvimento da aterosclerose. Quanto ao sexo 65,4\% eram do sexo masculino e 34,6\% do sexo feminino. Em relação à etnia 19,4\% se autodeclararam brancos, 46,7\% pardos e 33,9\% negros, assim como demonstra a Tabela 2.

Tabela 2. Características sociodemográficas dos pacientes HIV/AIDS em uso de TARV acompanhados nos serviços ambulatoriais, Aracaju, 2018.

\begin{tabular}{lccc}
\hline Variáveis & Total & Valor de p \\
\hline Faixa etária & & $1,26 \%$ \\
19 & 2 & $6,29 \%$ \\
$20-29$ & 10 & $22,64 \%$ \\
$30-39$ & 36 & $30,19 \%$ \\
$40-49$ & 48 & $27,04 \%$ \\
$50-59$ & 43 & $10,69 \%$ \\
$60-69$ & 17 & $1,26 \%$ \\
$70-79$ & 2 & $0,63 \%$ \\
80 & 1 & \\
Sexo & & $34,6 \%$ \\
Feminino & 55 & $65,4 \%$ \\
Masculino & 104 & $20,13 \%$ \\
Etnia & & $35,22 \%$ \\
Branco & 32 & $44,65 \%$ \\
Negro & 56 & $\mathbf{1 0 0 \%}$ \\
Pardo & 71 & $<01$ \\
\hline Total & $\mathbf{1 5 9}$ & $<001$ \\
\hline
\end{tabular}

Fonte: SICLOM/PMIST-AIDS-HV/REPVAS/DVAS/SMS-AJU.

Dessa amostra 23,9\% dos pacientes fizeram todos os exames de acompanhamento necessários para a análise da pesquisa, 59,1\% fizeram TE e ECO e 43,9\% fizeram TC. No período estudado segundo os critérios de definição de casos (Critério CDC Adaptado) estabelecida pelos Centers for Disease Control and Prevention (CDC), 22,64\% dos pacientes estavam com CD4 menor ou igual a 350 classificados como AIDS, 71,70\% como HIV e 5,66\% ainda não tinham realizado (Tabela 3).

Considerando a classe terapêutica do número de pacientes em uso de TARV, 44,66\% dos pacientes estavam ou fizeram uso de esquemas contendo IP e, quanto ao tempo de uso, 36,48\% há mais de 10 anos em TARV, tendo a amostra uma variação entre 15 dias e 32 anos. A duração média de uso da terapia foi de 72 meses (intervalo entre 1 a 300 meses), reforçando que o risco excessivo cardiovascular está relacionado à exposição, em longo prazo, os quais efeitos sinérgicos sugerem possíveis mecanismos celulares diretos pelos quais os IPs podem promover aterosclerose. Para as variáveis independentes (tratamento e tempo de uso) foi realizada análise não paramétrica (Tabela 3).

Tabela 3. Características clínicas dos pacientes HIV/AIDS em uso de TARV acompanhados nos serviços ambulatoriais, Aracaju, 2018.

\begin{tabular}{lccc}
\hline Variáveis & Total & \% & Valor de p \\
\hline CD4 & & & \\
$\leq 350$ & 36 & $22,64 \%$ & $<0,0001$ \\
$>350$ & 114 & $71,70 \%$ & \\
$\quad$ Não realizado & 9 & $5,66 \%$ & \\
Classe terapêutica & & & $<0,0001$
\end{tabular}




\begin{tabular}{lcc}
\hline & & \\
INTEGRASE (INI) + ITRN & 35 & $22,01 \%$ \\
IP + ITRN & 66 & $41,51 \%$ \\
IP + INTEGRASE (INI) & 4 & $2,52 \%$ \\
MARAVIROC + IP + INTEGRASE (INI) & 1 & $0,63 \%$ \\
Tempo de TARV & & \\
$\leq 2$ anos & 28 & $17,61 \%$ \\
$>2$ anos e $\leq 10$ anos & 73 & $45,91 \%$ \\
$>10$ anos & 58 & $36,48 \%$ \\
\hline Total & $\mathbf{1 5 9}$ & $\mathbf{1 0 0 \%}$ \\
\hline
\end{tabular}

Fonte: SICLOM/PMIST-AIDS-HV/REPVAS/DVAS/SMS-AJU.

A partir desses dados secundários oriundos dos prontuários dos pacientes referentes e calculados os EC, foram classificados pelo grau de calcificação coronariana definida a presença da aterosclerose com CAC > 0 (Agatston total e por segmentos, além de percentil quando a idade se adequar) na Tabela 4.

Tabela 4. Classificação dos valores de escore de cálcio de acordo com o grau de acometimento aterosclerótico.

\begin{tabular}{ccccc}
\hline \multicolumn{5}{c}{ Grau de Calcificação Coronariana } \\
\hline \multicolumn{5}{c}{ Valores Ajustados (Sexo e Idade) } \\
\hline Percentil & Grau de calcificação & $\mathbf{N}^{\mathbf{0}}$ pacientes & \% & Valor de p \\
\hline 0 & Ausência de calcificação & 53 & $68,83 \%$ \\
$0-25$ & Mínimo & 2 & $2,60 \%$ \\
$26-50$ & Leve & 3 & $3,90 \%$ & $<0,0001$ \\
$51-75$ & Moderado & 6 & $7,79 \%$ & $6,49 \%$ \\
$76-90$ & Severo & 5 & $10,39 \%$ \\
\hline 90 & Muito severo & 8 & $\mathbf{1 0 0 \%}$ \\
\hline
\end{tabular}

Fonte: HU-UFS/CEMAR.

Os pacientes foram estratificados pelos escores de Framingham, classificados até $10 \%$ como baixo risco, de 10 a $20 \%$ risco moderado e mais de $20 \%$ alto risco, como demonstrado na Tabela 5.

Tabela 5. Classificação risco pelos escores de Framingham, por sexo, Aracaju, 2018.

\begin{tabular}{cccccc}
\hline Risco em 10 anos & Feminino & \% & Masculino & \% & Valor de p \\
\hline Baixo Risco & 53 & $96,36 \%$ & 90 & $86,54 \%$ & \\
Médio Risco & 02 & $3,64 \%$ & 07 & $6,73 \%$ & $<, 0097$ \\
Alto Risco & 00 & $0,00 \%$ & 07 & $6,73 \%$ & \\
\hline Total & $\mathbf{5 5}$ & $\mathbf{1 0 0 \%}$ & $\mathbf{1 0 4}$ & $\mathbf{1 0 0 \%}$ \\
\hline
\end{tabular}

Fonte: HU-UFS/CEMAR

A presença de isquemia miocárdica está descrita na Tabela 6. Na análise das variáveis categóricas mostraram uma tendência de associar-se à aterosclerose, a hipertensão acometia 23,9\% dos pacientes, 36\% apresentavam colesterol total igual ou superior a $200 \mathrm{mg} / \mathrm{dl}, 29,56 \%$ tinham níveis séricos de triglicérides superior a 150mg/dl, 25,16\% apresentavam dislipidemia e apenas $12,58 \%$ eram tabagistas (Tabela 6). 
Tabela 6. Comorbidades dos pacientes HIV/AIDS em uso de TARV, Aracaju, 2018.

\begin{tabular}{|c|c|c|c|}
\hline Comorbidades & Total & $\%$ & Valor de p \\
\hline \multicolumn{4}{|c|}{ Isquemia miocárdica } \\
\hline $1(\mathrm{Sim})$ & 11 & $10,78 \%$ & \multirow[t]{2}{*}{$<0,0001$} \\
\hline 2 (Não) & 91 & $89,22 \%$ & \\
\hline \multicolumn{4}{|c|}{ Hipertensão arterial } \\
\hline Não & 125 & $78,61 \%$ & \multirow{2}{*}{$<0,0001$} \\
\hline Sim & 34 & $21,39 \%$ & \\
\hline \multicolumn{4}{|l|}{ Colesterol total } \\
\hline$<160$ & 36 & $22,64 \%$ & \multirow[t]{6}{*}{$<0,0001$} \\
\hline $160-199$ & 44 & $27,67 \%$ & \\
\hline $200-239$ & 25 & $15,72 \%$ & \\
\hline $240-279$ & 12 & $7,55 \%$ & \\
\hline$>280$ & 8 & $5,03 \%$ & \\
\hline Sem exame & 34 & $21,39 \%$ & \\
\hline \multicolumn{4}{|l|}{ Triglicérides } \\
\hline Não & 68 & $42,76 \%$ & \multirow[t]{3}{*}{0,0397} \\
\hline Sim & 47 & $29,56 \%$ & \\
\hline Sem exame & 44 & $27,68 \%$ & \\
\hline \multicolumn{4}{|l|}{ Dislipidemia } \\
\hline Não & 115 & $72,33 \%$ & \multirow[t]{3}{*}{$<0,0001$} \\
\hline Sim & 40 & $25,16 \%$ & \\
\hline Sem exame & 4 & $2,51 \%$ & \\
\hline \multicolumn{4}{|l|}{ Tabagismo } \\
\hline Não & 139 & $87,42 \%$ & \multirow[t]{2}{*}{$<0,0001$} \\
\hline Sim & 20 & $12,58 \%$ & \\
\hline
\end{tabular}

Fonte: HU-UFS/CEMAR

Os valores de p foram considerados estatisticamente significativos em 100\% das variáveis analisadas.

\section{Discussão}

A aterosclerose coronariana detectada pelo EC e avaliada pela TC cardíaca foi encontrada em 24 pacientes $(31,17 \%)$ e o tempo de uso da TARV teve significância. Esse achado está de acordo com a literatura, onde números semelhantes são apontados (Kingsley et al., 2008). Talwani et al. (2002) haviam exposto que o EC nas pessoas vivendo com HIV/AIDS em uso de TARV por, pelo menos, seis meses não era significativamente diferente das pessoas não portadoras do vírus. Quanto a idade dos pacientes em estudo (média de 46 anos) também é possível expor que comparando a estudos Kingsley et al. (2008) encontraram um EC positivo em $12 \%$ dos pacientes soropositivos com menos de 45 anos de idade e em $23 \%$ dos pacientes com idade entre 40 e 45 anos. No entanto, esse estudo mostrou um percentual maior de aterosclerose coronariana em pacientes em TARV, quando todas as faixas etárias foram incluídas, em $\geq 50$ anos. A idade teve influência direta no desenvolvimento da aterosclerose e pode-se supor que a ausência da prevalência de aterosclerose em relação à população estudada deve-se a ter uma média da faixa etária de população mais jovem. A idade, portanto, é um dos principais fatores de risco para o desenvolvimento da aterosclerose em pessoas com AIDS (Hulten et al., 2009) semelhantemente à população em geral (Ho et al., 2008).

A duração média do uso da TARV nessa população foi de 72 meses, provavelmente não foi necessário para o desenvolvimento da aterosclerose. O estudo de Kingsley et al. (2008) também demonstrou que a aterosclerose detectada pelo EC foi significativamente maior em pacientes que estiveram por, pelo menos, oito anos, em TARV que é o período para se 
desenvolver a doença, comprovando na população em estudo em que 36,48\% dos pacientes que apresentaram a aterosclerose estavam em uso de TARV em média há mais de 10 anos. Os dados negativos sobre a prevalência de aterosclerose em indivíduos com HIV em TARV por, pelo menos, dois anos são importantes na prática clínica diária, sugerindo que um período mais longo da TARV é necessário para a detecção da aterosclerose, mesmo quando determinados métodos, como a TC cardíaca, são empregados. Esses dados também foram confirmados pelo estudo de Kingsley et al. (2008).

Sabe-se que das classes terapêuticas de antirretrovirais o uso dos IP estão mais relacionados à intolerância à glicose e síndrome metabólica (SM) e a alterações lipídicas,. Johnsen et al. (2006) demonstraram aumento no espessamento da carótida de pacientes em uso de IP, onde sugere-se que há evidências da indução aterosclerótica potencial e subsequente da doença cardiovascular. A relação entre a prevalência da aterosclerose e o tipo de TARV (com ou sem IP) foi o objeto de análise do presente estudo e observou-se diferença significativa na prevalência da aterosclerose entre as classes de fármacos envolvidas. Vale ressaltar que, na maioria dos estudos que encontraram presença de maior grau de aterosclerose e eventos cardiovasculares, tais como isquemia miocárdica em pacientes, foram sob uso de IP (Kingsley et al., 2008; Lai et al., 2003). 44,66\% da população do presente estudo estiveram em uso de IP. Da amostra de pacientes que tiveram a detecção da aterosclerose, 50\% desse percentual estavam em uso de IP. Mesmo na classe dos IP, como Lopinavir e Atazanavir, novos medicamentos antirretrovirais como Darunavir que apresentam perfil lipídico mais seguro em comparação aos semelhantes têm sido utilizados nos últimos anos (Colafigli et al., 2008). Os resultados de estudos mostram uma associação entre TARV contendo IP, embora sejam drogas potentes para melhorar os resultados clínicos de pacientes com HIV, evitando replicação viral e diminuindo a carga viral com fortes efeitos adversos, reforçando que o risco excessivo cardiovascular está relacionado à exposição há longo prazo, pelos quais os IPs podem promover aterosclerose e apresenta como principais reações adversas a toxicidade metabólica; lipodistrofia, dislipidemia, hiperglicemia, resistência a insulina e diabetes, variáveis essas que predispõem a DAC. A análise mostra que os resultados foram influenciados pelo desenho do estudo e pela idade. Este estudo não revelou diferenças significativas em relação ao desenvolvimento de aterosclerose em relação ao sexo ou à presença da maioria dos fatores de risco.

No estudo 23,9\% dos pacientes tinham hipertensão e 29,56\% diabetes, 40,8\% com triglicérides fora do padrão, 34,9\% apresentavam alteração no colesterol e apenas $12,58 \%$ eram tabagistas. Todos esses fatores levam à maior prevalência e intensidade de aterosclerose em pacientes com AIDS. A aterosclerose em pacientes soropositivos é o resultado de diferentes fatores, dos quais o mais importante é o metabolismo lipídico (Grinspoon, 2005). Há uma redução primária do colesterol HDL resultante da ativação imune pelo HIV e do estado de hipercatabolismo da doença (Oh \& Hegele, 2007). A TARV tem um efeito ainda maior sobre o metabolismo lipídico. Diferentes esquemas antirretrovirais podem aumentar a produção de VLDL, potencializando a quebra de lipoproteínas ricas em triglicerídeos, levando à maior concentração sérica. Além do efeito da TARV no metabolismo lipídico, seu efeito sobre a resistência à insulina e sobre a SM também é importante (Grinspoon, 2005).

O aumento em pacientes com AIDS do risco de hipertensão arterial sistêmica é fator de risco cardiovascular adicional para esse grupo de pacientes e também está descrito em Arruda Júnior et al., 2010, mostrando a influência direta desses fatores na aterosclerose detectada em função do Ecocardiograma. Em relação aos fatores de risco tradicionais não se observou associação da aterosclerose. Sabe-se que os escores de riscos tradicionais podem subestimar o risco cardiovascular de PVHIV, o que tem direcionado os estudos científicos para análise de comorbidades e exames não invasivos para pesquisa de aterosclerose nesses pacientes, EC arterial mensurado por TC ou ressonância magnética e angiotomografia de placas de aterosclerose não calcificadas.

A maioria de nossos resultados mostrou uma tendência para maior prevalência de fatores de risco tradicionais na população com aterosclerose, apesar da falta de valores significativos.

O escore de Framingham foi determinado para essa população, a fim de estimar o risco cardiovascular em dez anos. 
Um total de 96,36\% dos pacientes do sexo feminino e 86,54\% do sexo masculino teve um escore de Framingham baixo, principalmente em razão da menor idade dos pacientes no estudo. Não houve associação significativa entre um escore de Framingham mais elevado e um EC positivo. Neste estudo, pode-se supor que o escore de Framingham não representa a melhor ferramenta para determinar o risco cardiovascular na população específica estudada. No entanto, embora o cálculo da amostra em um estudo de prevalência é de fundamental importância, este estudo, por se tratar de natureza descritiva, visou a determinar a prevalência da aterosclerose nesses pacientes, a partir dos fatores de risco associados. Devendo a partir dos dados apresentados incentivarem os pesquisadores a realizar estudos mais amplos, a fim de testar as hipóteses aqui levantadas.

As comorbidades metabólicas aqui descritas, a saber, dislipidemia, HAS, DM ou resistência insulínica e o excesso de peso ou obesidade, quando associadas constituem a chamada SM (Monte et al., 2019; Rogalska-Płońska et al., 2018). Assim aferindo para cada comorbidade isolada, também há estudos que demonstram maior prevalência de SM em pessoas que vivem com o HIV (Rogalska-Płońska et al., 2018). Contudo, há significativa variabilidade de prevalência e de acordo com o esquema terapêutico disponível para cada amostra de pacientes avaliadas, pode causar diferentes efeitos metabólicos com frequência também variável (Rogalska-Płońska et al., 2018).

Este estudo foi considerado de extrema importância clínica, uma vez que saber o melhor momento para fazer o diagnóstico evita gastos desnecessários para o sistema de saúde e nesse sentido, sugere-se a criação de ferramentas específicas para avaliação cardiovascular em PVHIV, adicionando status imunológico e marcadores laboratoriais inflamatórios aos fatores de risco tradicionais. (Paula, Falcão \& Pacheco, 2013).

Como limitação desta pesquisa é possível citar: 1) A presença de diversos confundidores (a exemplo da dislipidemia que tem a própria TARV como possível causa) que trouxe dificuldades em definir a gênese da aterosclerose nesta população. De qualquer forma tal limitação vem sendo apontada em outros estudos na área, sendo argumento comum a complexa interação de fatores na citada gênese; e 2) A dificuldade de inclusão dos pacientes por ser demanda espontânea nos ambulatórios - tanto pela disponibilidade, quanto por impossibilidade causada pelo estado de saúde e por constar de etapas sucessivas, de questionário a exames, puderam-se enfrentar algumas resistências. Para contornar estas situações, houve o adequado acolhimento dos sujeitos da pesquisa, com esclarecimento dos benefícios para os mesmos, sobretudo do ponto de vista de prevenção. Este estudo teve uma amostra de $n=159$ pacientes, considerando o Intervalo de confiança de $95 \%$ e uma diferença aceitável de $10 \%$, a amostra desta pesquisa teria um $\mathrm{n}=94$, mas pelas limitações que o próprio estudo traz como possível dificuldade de adesão pelos pacientes durante todo o estudo e não sendo tido como oneração de gasto foi possível atingir um número maior de cadastro.

\section{Conclusão}

Este estudo mostrou uma baixa prevalência de aterosclerose em pacientes com HIV/AIDS utilizando-se a análise da classificação de risco pelos escores de Framingham e pelo grau de calcificação coronariana definida por Agatston e quanto à relação do uso de TARV tem-se entre os fatores de risco avaliados, a idade, o tempo de uso e tipos de esquemas associados ao desenvolvimento da aterosclerose.

Assim, apesar das evidências científicas comprovadas da relação da TARV para a aterosclerose em pacientes com HIV/AIDS, o estudo revelou que um tempo prolongado de exposição a esse tratamento é necessário para a aterosclerose ser detectada por meio de TC cardíaca. E a prevalência da aterosclerose nos pacientes que vivem com HIV documentada pelo EC em torno de $40 \%$, representou $31,17 \%$ da amostra estudada com comprometimento aterosclerótico.

É importante realizar estudos em nível local a fim de contemplar peculiaridades da citada população que possam influenciar nos desfechos. O conhecimento advindo desta pesquisa possibilitará ainda uma melhor compreensão das interaç̃oes entre o HIV, os esquemas antirretrovirais e o paciente, podendo modificar a indicação dos mesmos em pacientes de maior 
risco.

\section{Referências}

Arruda Júnior, E. R., Lacerda, H. R., Moura, L. C. R. V., Albuquerque, M. F. P. M., Miranda Filho, D. B., Diniz, G. T. N., Albuquerque, V. M. G., Amaral, J. C. Z., Monteiro, V. S., \& Ximenes, R. A. A. (2010). Perfil dos pacientes com hipertensão arterial sistêmica incluídos em uma coorte com HIV/Aids em Pernambuco, Brasil. Arquivos Brasileiros de Cardiologia, 95(5), 640-647. https://doi.org/10.1590/S0066-782X2010005000138

Brasil. (2020). Ministério da Saúde. Departamento de Doenças de Condições Crônicas e Infecções Sexualmente Transmíssiveis. (2020). Boletim epidemiológico - Brasília, 2020. aids.gov.br/pt-br/pub/2020/boletim-epidemiologico-hivaids-2020

Colafigli, M., Di Giambenedetto, S., Bracciale, L., Tamburrini, E., Cauda, R., \& Luca, A. (2008). Cardiovascular risk score change in HIV-1-infected patients switched to an atazanavir-based combination antiretroviral regimen. HIV medicine, 9(3), 172-179. https://doi.org/10.1111/j.1468-1293.2007.00541.x

Grinspoon, S. K. (2005). Metabolic syndrome and cardiovascular disease in patients with human immunodeficiency virus. The American journal of medicine, 118(Supp1 2), 23S-28S. https://doi.org/10.1016/j.amjmed.2005.01.047

Oh, J., \& Hegele, R. A. (2007). HIV- associated dyslipidaemia: pathogenesis and treatment. The Lancet infectious diseases, 7(12), 787-796. https://doi.org/10.1016/s1473-3099(07)70287-6

Ho, J. S., Fitzgerald, S. J., Stolfus, L. L., Wade, W. A., Reinhardt, D. B., Barlow, C. E., \& Cannaday, J. J. (2008). Relation of a coronary artery calcium score higher than 400 to coronary stenoses detected using multidetector computed tomography and to traditional cardiovascular risk factors. The American journal of cardiology, 101(10), 1444-1447. https://doi.org/10.1016/j.amjcard.2008.01.022

Hulten, E., Mitchell, J., Scally, J., Gibbs, B., \& Villines, T. C. (2009). HIV positivity, protease inhibitor exposure and subclinical atherosclerosis: a systematic review and meta-analysis of observational studies. Heart, 95(22), 1826-1835. https://doi.org/10.1136/hrt.2009.177774

Johnsen, S., Dolan, S. E., Fitch, K. V., Kanter, J. R., Hemphill, L. C., Connelly, J. M., Lees, R. S., Lee, H., \& Grinspoon, S. (2006). Carotid intimal medial thickness in Human Immunodeficiency Virus-infected women: effects of protease inhibitor use, cardiac risk factors, and the metabolic syndrome. The journal of clinical endocrinology and metabolism, 91(12), 4916-4924. https://doi.org/10.1210/jc.2006-1140

Kingsley, L. A., Cuervo-Rojas, J., Muñoz, A., Palella, F. J., Post, W., Witt, M. D., Budoff, M., \& Kuller, L. (2008). Subclinical coronary atherosclerosis, HIV infection and antiretroviral therapy: Multicenter AIDS Cohort Study. AIDS, 2008, 22(13), 1589-1599. https://dx.doi.org/10.1097\%2FQAD.0b013e328306a6c5

Lai, S., Lai, H., Celentano, D. D., Vlahov, D., Ren, S., Margolick, J., Lima, J. A. C., \& Bartlett, J. G. (2003). Factors associated with accelerated atherosclerosis in HIV-1 infected persons treated with protease inhibitors. AIDS Patient Care STDs, 17(5), 211-219. https://doi.org/10.1089/108729103321655863

Monte, I. P., França, S. L., Vasconcelos, R. N. O., \& Vieira, J. R. S. (2019). Comparação entre quatro diferentes critérios de diagnóstico de síndrome metabólica em indivíduos do Arquipélago do Marajó (Pará, Brasil). Revista da Associação Brasileira de Nutrição - RASBRAN, 10(1), 96-102. https://www.rasbran.com.br/rasbran/article/view/1242

Paula, A. A., Falcão, M. C. N., \& Pacheco, A. G. (2013). Metabolic syndrome in HIV-infected individuals: underlying mechanisms and epidemiological aspects. AIDS Research and Therapy, 10(1). https://dx.doi.org/10.1186\%2F1742-6405-10-32

Rogalska-Płońska, M., Grzeszczuk, A., Rogalski, P., Łucejko, M., \& Flisiak, R. (2018). Metabolic syndrome in HIV infected adults in Poland. Kardiologia Polska, 76(3), 548-553. https://doi.org/10.5603/KP.a2017.0249

Saddi, P., \& Giraldez, V. Z. R. (2009). Alterações metabólicas e risco cardiovascular associados ao HIV. http://www.fleury.com.br/medicos/educacaomedica/artigos/Pages/alteracoes-metabolicas-e-risco-cardiovascular-associados-ao-hiv.aspx

Talwani, R., Falusi, O. M., Leon, C. F. M., Nerad, J. L., Rich, S., Proia, L. A., Sha, B. E., Smith, K. Y., \& Kessler, H. A. (2002). Electron beam computed tomography for assessment of coronary artery disease in HIV-infected men receiving antiretroviral therapy. Journal of acquired immune deficiency syndromes, 30(2), 191-195. https://doi.org/10.1097/00042560-200206010-00008

Thienemann, F., Sliwa, K., \& Rockstroh, J. K. (2013). HIV and the heart: the impact of antiretroviral therapy: a global perspective. European heart journal, 34(46), 3538-3546. https://doi.org/10.1093/eurheartj/eht388

UNAIDS. (2010). Global report: UNAIDS report on the global AIDS epidemic 2010.

https://files.unaids.org/en/media/unaids/contentassets/documents/unaidspublication/2010/20101123_globalreport_en\%5b1\%5d.pdf

UNAIDS. (2011). UNAIDS 2011 World AIDS Day report.

https://www.unaids.org/en/resources/documents/2011/20111121_JC2216_WorldAIDSday_report_2011

WHO. (2016). Global health sector strategy on HIV 2016-2021. Towards ending AIDS. World Health Organization.

https://apps.who.int/iris/handle/10665/246178 